A C G

publications

Org. Commun. 13:1 (2020) 19-27

organic

communications

\title{
Synthesis and characterization of some novel fluoro and methoxy substituted chalcone derivatives via nucleophilic aromatic substitution
}

\author{
Derya Aktaş Anıl ${ }^{1}{ }^{1}$ and M. Fatih Polat (C) 2,* \\ ${ }^{I}$ Department of Chemistry and Chemical Process Technologies, Erzurum Vocational School, Atatürk \\ University, 25240, Erzurum, Türkiye \\ ${ }^{2}$ Department of Pharmaceutical Basic Sciences, Faculty of Pharmacy, Erzincan Binali Yıldırım \\ University, 24100, Erzincan, Türkiye
}

(Received February 17,2020 ; Revised March 17, 202; Accepted March 21, 2020 )

\begin{abstract}
In this study, 2',4',6'-Trimethoxyacetophenone was reacted with various fluorine-substituted benzaldehydes in the presence of an aqueous solution of 50\%-potassium hydroxide, and new chalcone derivatives in the structure of 1,3-diaryl-2-propenone comprising both fluorine and methoxy groups in the aromatic B ring were obtained in high yields under mild conditions. In base-catalyzed Claisen-Schmidt condensations, $\mathrm{MeOH}$ is usually used as solvent. In the condensation reactions performed with mono-fluorine-substituted benzaldehydes in $\mathrm{MeOH}$, the corresponding fluorine-substituted chalcone derivatives in the B ring were synthesized in high yield. On the other hand, when di- and tri-fluorine-substituted benzaldehydes were used, it was determined that a nucleophilic aromatic substitution $\left(S_{N} A r\right)$ occurred in the reaction condition and the condensation yield decreased. Consequently, fluorine-methoxy substituted chalcone derivatives were isolated by substitution of fluorine and methoxy group in para position in the aromatic B ring. On the other hand, it was found that when THF was used as the reaction solvent, chalcone derivatives carrying only fluorine atoms could be obtained with excellent chemical selectivity without any competitive aromatic nucleophilic substitution $\left(\mathrm{S}_{\mathrm{N}} \mathrm{Ar}\right)$ in the condition. Particularly in the synthesis studies of such fluorine-substituted chalcones, it was determined that the Claisen-Schmidt condensation ratio is dependent on the effect of the solvent in addition to steric and electronic factors.
\end{abstract}

Keywords: Fluorine; methoxy; chalcone; synthesis. (C2020 ACG Publication. All right reserved.

\section{Introduction}

Over the recent years, fluorine chemistry has played a significant role in various technological developments including the fields of fluoropolymers, materials science, pharmaceuticals and agricultural products. ${ }^{1}$ Having overcome the synthetic difficulties encountered in medicinal chemistry as well as structure-reactivity relationships observed in fluorine-containing compounds has increased the interest in studies in this field. ${ }^{2}$ In particular, the chemical properties that the $\mathrm{C}-\mathrm{F}$ bond, the basic building block of organofluorine chemistry, exhibits as a substituent as well as type of structures and positions are carefully examined. ${ }^{3}$ Arenes containing fluorine in the aromatic benzene ring, which can be considered as the most important of these structures, are more suitable than hydrogen-based arenes against nucleophilic attacks owing specifically to their electronic properties. Therefore, fluorine-substituted arenes offer new

\footnotetext{
* Corresponding author: E-Mail: fpolat@erzincan.edu.tr, Phone: + 90 4462245344; Fax: + 904462245343.
} 
approaches in the synthesis of compounds that may be medicinally important due to nucleophilic aromatic substitution reactions. ${ }^{4}$

When the mechanisms of nucleophilic substitution reactions on fluorine-containing aromatic compounds are examined, especially when all positions are fluorine-substituted, it is assumed that the nucleophile first develops into a transition complex as a result of a combination and then proceeds with the elimination of the fluoride anion. ${ }^{5}$ In this resonance stable transition complex, which is known as the Meisenheimer complex, a new carbon-nucleophile bond is formed. This step quickly occurs as the ring will then start to aromatize by elimination of fluoride anion as an easily separated group. What is important here is that there are many fluorine substituents as well as some electron withdrawing groups in the aromatic ring. ${ }^{6}$ Using polyfluoro-aromatic compounds that comprise a large number of fluorine in the aromatic ring, the fluorine ion at the ortho, meta, or para position is expected to be replaced by the nucleophile. When the reaction mechanism is examined, the negatively charged transition complex formed at the intermediate stage should be stabilized. This stability is achieved by the combined inductive effects of fluorine atoms in the ring. The nucleophilic attack occurs in the ortho and para positions as this inductive effect prevents $\mathrm{p}-\pi$ overlap of $\mathrm{sp}^{2}$ hybridized carbon-bound fluorine. The general stabilization of the negatively charged intermediate product is considered to be the case where fluorine in the para position is least effective. Therefore, the nucleophilic attack usually occurs from the para position in polyfluorinated arenes (Scheme 1). ${ }^{7}$

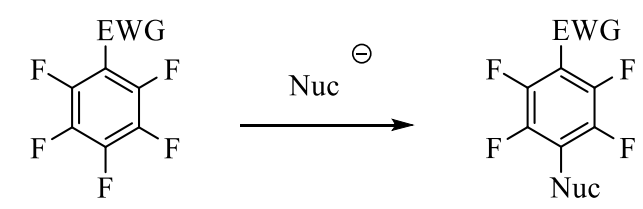

Scheme 1. Nucleophilic aromatic substitution reaction of polifluoro arenes. ${ }^{7}$

As can be understood from here, highly fluorinated aromatic compounds are used in various nucleophilic aromatic displacement reactions. To increase the efficiency of the nucleophilic aromatic substitution, Meisenheimer complexes are applied with strong electron withdrawing groups such as nitro, as well as by preferring structures comprising plenty of fluorine atoms. ${ }^{8}$

In this study, in addition to the synthesis of chalcone derivatives containing fluorine in their structures, chalcone derived structures containing both fluorine and methoxy groups in different positions were effectively and quickly obtained through nucleophilic aromatic substitution reaction $\left(\mathrm{S}_{\mathrm{N}} \mathrm{Ar}\right)$, which is predicted to have occurred in the aromatic ring.

In recent years, there have been several drug compounds that contain fluorine, which were medically approved by FDA which are of great importance in terms of both organic and medicinal chemistry. ${ }^{9}$ Compounds containing the $\mathrm{C}-\mathrm{F}$ bond have become more attractive to the researchers due to small atomic radius, high electronegativity, nuclear spin of $1 / 2$, and low polarization of the fluorine atom. In addition, it is reported that compounds that comprise fluorine atoms generally bind to target molecules more easily due to their higher metabolic stability. ${ }^{10}$ Again, because of the high lipophilic properties of these molecules and the ease of their membrane penetration, the C-F bond is biologically activated compared to the $\mathrm{C}-\mathrm{H}$ and $\mathrm{C}-\mathrm{O}$ bonds. ${ }^{11}$

Chalcones are compounds that have 1,3 diaryl-2-propen-1-one structure, that comprise the $\alpha, \beta$ unsaturated ketone group and that form a subgroup of the flavonoid family. ${ }^{12}$ Chalcones, which can be obtained both naturally and synthetically, have many biological properties such as being antibacterial, antiulcer, antiprotozoal, anthelmintic, insecticidal, amoebicidal, immunosuppressive, cytotoxic, $\alpha$ glucosidase and anticancer. ${ }^{13-15}$ On the other hand, chalcones have been reported to be highly effective, especially in the anticancer armamentarium thanks to the different substituents (methoxy, amino, nitro, halogen, and hydroxyl groups) in different positions on the two aromatic rings they have. ${ }^{16}$ The chalcones show excellent cytotoxic activity not only in blocking the cellular division process but also in different cell death pathways. ${ }^{17}$

Based on these issues, it was aimed in this study to obtain compounds that contain methoxy and fluorine in aromatic rings that are present in chalcone structure, in high yield, one-pot synthesis. 


\section{Experimental}

\subsection{General}

All synthesized chalcone structures were verified using spectral techniques such as FT-IR, ${ }^{1} \mathrm{H}-$ NMR, ${ }^{13} \mathrm{C}-\mathrm{NMR}$ and Elemental analysis. The ${ }^{1} \mathrm{H}$-NMR and ${ }^{13} \mathrm{C}-\mathrm{NMR}$ spectra of chalcone compounds were measured using Varian $400 \mathrm{MHz}$ and Bruker $400 \mathrm{MHz}$ spectrometry in Chloroform-d at ambient temperature. Fourier transformed infrared spectroscopy (FT-IR) spectra were recorded in the wavenumber range of 400-4000 $\mathrm{cm}^{-1}$ using Perkin Elmer Spectrum One FT-IR spectrometer. The melting points were measured using a Gallenkamp melting point apparatus. The elemental analysis results were confirmed using a Leco CHNS-932 device. Commercially purchased solvents and chemicals were used.

\subsection{Chemistry}

\subsubsection{General Procedure for Preparation of Compounds $(7,8,10,12,14)$}

To a solution of $2^{\prime}, 4^{\prime}, 6^{\prime}$-trimethoxy acetophenone, $(0.48 \mathrm{mmol})$ in $\mathrm{MeOH}(10 \mathrm{~mL})$ benzaldehyde derivatives $2, \mathbf{3}, \mathbf{4}, \mathbf{5}, \mathbf{6}(0.77 \mathrm{mmol})$ and $50 \% \mathrm{KOH}$ solution $(0.13 \mathrm{~mL})$ was added and resultant mixture was sequentially stirred overnight at room temperature. The solvent was evaporated in vacuum. Crude material was extracted with $\mathrm{NH}_{4} \mathrm{Cl}$ solution $(10 \mathrm{~mL})$ and dichloromethane (DCM) $(15 \mathrm{~mL}$ x 3 ). The combined extracts were dried over $\mathrm{Na}_{2} \mathrm{SO}_{4}$. The solvent was removed in vacuum.

(E)-3-(2-Fluorophenyl)-1-(2,4,6-trimethoxyphenyl)prop-2-en-1-one (7): Crude product was precipitated with $(\mathrm{DCM} / \mathrm{Hexane})$. Final product yellow solid; yield 92\%. $\mathrm{R}_{f}=($ EtOAc/Hexane $30: 70)=0.23 ; \mathrm{m} . \mathrm{p}=$ 103-106 ${ }^{\circ} \mathrm{C}$; ${ }^{1} \mathrm{H}-\mathrm{NMR}\left(400 \mathrm{MHz}, \mathrm{CDCl}_{3}\right) \delta 7.59-7.50(\mathrm{~m}, 2 \mathrm{H}), 7.36-7.30(\mathrm{~m}, 1 \mathrm{H}), 7.17-7.13(\mathrm{~m}$, $1 \mathrm{H}), 7.09-7.01(\mathrm{~m}, 2 \mathrm{H}), 6.16(\mathrm{~s}, 2 \mathrm{H}), 3.86(\mathrm{~s}, 3 \mathrm{H}), 3.78(\mathrm{~s}, 6 \mathrm{H}) ;{ }^{13} \mathrm{C}-\mathrm{NMR}\left(100 \mathrm{MHz}, \mathrm{CDCl}_{3}\right) \delta 194.2$, $161.1\left(\mathrm{~d}, \mathrm{C}-16, J_{C F}=252.1 \mathrm{~Hz}\right), 162.6,159.1,136.2\left(\mathrm{~d}, \mathrm{C}-10, J_{C F}=3.3 \mathrm{~Hz}\right), 131.5\left(\mathrm{~d}, \mathrm{C}-14, J_{C F}=8.7\right.$ $\mathrm{Hz}), 131.1\left(\mathrm{~d}, \mathrm{C}-12, J_{C F}=5.7 \mathrm{~Hz}\right), 128.9\left(\mathrm{~d}, \mathrm{C}-8, J_{C F}=2.9 \mathrm{~Hz}\right), 124.4\left(\mathrm{~d}, \mathrm{C}-13, J_{C F}=3.6 \mathrm{~Hz}\right), 123.2(\mathrm{~d}$, $\left.\mathrm{C}-11, J_{C F}=11.8 \mathrm{~Hz}\right), 116.1\left(\mathrm{~d}, \mathrm{C}-15, J_{C F}=21.6 \mathrm{~Hz}\right), 111.6,90.7,55.9,55.5 ; \mathrm{IR}\left(\mathrm{KBr}, \mathrm{cm}^{-1}\right) \operatorname{Vmax}: 2941$, 1607, 1458, 1269, 1228; Elemental Analysis Anal. calcd for $\mathrm{C}_{18} \mathrm{H}_{17} \mathrm{FO}_{4}$ : C, 68.35; H, 5.42; Found: C, $68.09 ; \mathrm{H}, 5.51$.

(E)-3-(4-Fluorophenyl)-1-(2,4,6-trimethoxyphenyl)prop-2-en-1-one (8): Crude product was precipitated with $(\mathrm{DCM} /$ Hexane $)$. Final product yellow solid; yield $80 \% ; \mathrm{R}_{f}=(\mathrm{EtOAc} / \mathrm{Hexane} 30: 70)=0.27$; m.p. $122-123{ }^{\circ} \mathrm{C}$; ${ }^{1} \mathrm{H}-\mathrm{NMR}\left(400 \mathrm{MHz}, \mathrm{CDCl}_{3}\right) \delta 7.52-7.48(\mathrm{~m}, 2 \mathrm{H}), 7.32(\mathrm{~d}, 1 \mathrm{H}, \mathrm{B}$ part of AB system, $J=$ $16 \mathrm{~Hz}), 7.07-7.01(\mathrm{~m}, 2 \mathrm{H}), 6.87(\mathrm{~d}, 1 \mathrm{H}$, A part of AB system, $J=16 \mathrm{~Hz}), 6.15(\mathrm{~s}, 2 \mathrm{H}), 3.84(\mathrm{~s}, 3 \mathrm{H}), 3.76$ $(\mathrm{s}, 6 \mathrm{H}) ;{ }^{13} \mathrm{C}-\mathrm{NMR}\left(100 \mathrm{MHz}, \mathrm{CDCl}_{3}\right) \delta 194.1,164.0\left(\mathrm{~d}, \mathrm{C}-20, J_{C F}=249.8 \mathrm{~Hz}\right), 162.7,159.1,142.8,131.5$, $130.4\left(\mathrm{~d}, \mathrm{C}-18, J_{C F}=8.4 \mathrm{~Hz}\right), 129.0,116.1\left(\mathrm{~d}, \mathrm{C}-19, J_{C F}=21.7 \mathrm{~Hz}\right), 111.9,90.9,56.1,55.7$; IR $\left(\mathrm{KBr}, \mathrm{cm}^{-}\right.$ 1) Vmax: 3502, 2941, 2841, 1651, 1599; Elemental Analysis Anal. calcd for $\mathrm{C}_{18} \mathrm{H}_{17} \mathrm{FO}_{4}$ : C, 68.35; H, 5.42; Found: C, 68,16; H, 5.38.

(E)-3-(2-Fluoro-4-methoxyphenyl)-1-(2,4,6-trimethoxyphenyl)prop-2-en-1-one (10): Residue was purified via flash chromatography over silica gel using gradient elution with (EtOAc/Hexane). Final product yellow solid; yield 50\%; $\mathrm{R}_{f}=($ EtOAc/Hexane $40: 60)=0,5 ;$ m.p. $134-136{ }^{\circ} \mathrm{C} ;{ }^{1} \mathrm{H}-\mathrm{NMR}(400$ $\left.\mathrm{MHz}, \mathrm{CDCl}_{3}\right) \delta 7.48(\mathrm{~m}, 2 \mathrm{H}), 6.92(\mathrm{~d}, J=16.2 \mathrm{~Hz}, 1 \mathrm{H}), 6.70(\mathrm{~d}, J=8.6 \mathrm{~Hz}, 1 \mathrm{H}), 6.60(\mathrm{~d}, J=12.2 \mathrm{~Hz}$, $1 \mathrm{H}), 6.15(\mathrm{~s}, 2 \mathrm{H}), 3.85(\mathrm{~s}, 3 \mathrm{H}), 3.81(\mathrm{~s}, 3 \mathrm{H}), 3.76(\mathrm{~s}, 6 \mathrm{H}) ;{ }^{13} \mathrm{C}-\mathrm{NMR}\left(100 \mathrm{MHz}, \mathrm{CDCl}_{3}\right) \delta 195.2,164.4$ $\left(\mathrm{dd}, \mathrm{C}-14, J_{C F}=211.9,11.9 \mathrm{~Hz}\right), 164.1,161.7\left(\mathrm{dd}, \mathrm{C}-16, J_{C F}=215.5,11.7 \mathrm{~Hz}\right), 160.4,136.4,132.1,131.5$ $\left(\mathrm{d}, \mathrm{C}-12, J_{C F}=9,9 \mathrm{~Hz}\right), 121.0\left(\mathrm{~d}, \mathrm{C}-10, J_{C F}=11.5 \mathrm{~Hz}\right), 113.4\left(\mathrm{~d}, \mathrm{C}-13, J_{C F}=21.7 \mathrm{~Hz}\right), 112.9,105.9(\mathrm{~d}$, $\left.\mathrm{C}-15, J_{C F}=25.3,25.3 \mathrm{~Hz}\right), 92.2,57.3,57.1,56.9 . \mathrm{IR}\left(\mathrm{KBr}, \mathrm{cm}^{-1}\right)$ Vmax: $2941,1647,1605,1266,1156$, 1127; Elemental Analysis Anal. calcd for $\mathrm{C}_{19} \mathrm{H}_{19} \mathrm{FO}_{5}$ : C, 65.89; H, 5.53; Found: C, 65.53; H, 5.68.

(E)-3-(3-Fluoro-4-methoxyphenyl)-1-(2,4,6-trimethoxyphenyl)prop-2-en-1-one (12): Residue was purified via flash chromatography over silica gel using gradient elution with (EtOAc/Hexane). Final product yellow solid; yield 70\%; $\mathrm{R}_{f}=\left(\right.$ EtOAc/Hexane 40:60) $=0,46 ;$ m.p. $139-141{ }^{\circ} \mathrm{C} ;{ }^{1} \mathrm{H}-\mathrm{NMR}(400$ $\left.\mathrm{MHz}, \mathrm{CDCl}_{3}\right) \delta 7.30-7.19(\mathrm{~m}, 3 \mathrm{H}), 6.97-6.88(\mathrm{~m}, 1 \mathrm{H}), 6.81(\mathrm{~d}, J=16.0 \mathrm{~Hz}, 1 \mathrm{H}), 6.15(\mathrm{~s}, 2 \mathrm{H}), 3.89(\mathrm{~s}$, $3 \mathrm{H}), 3.84(\mathrm{~s}, 3 \mathrm{H}), 3.75(\mathrm{~s}, 6 \mathrm{H}) ;{ }^{13} \mathrm{C}-\mathrm{NMR}\left(100 \mathrm{MHz}, \mathrm{CDCl}_{3}\right) \delta 195.3,163.9,160.3,153.8(\mathrm{~d}, \mathrm{C}-15, J C F$ 
$=245.2 \mathrm{~Hz}), 150.8(\mathrm{C}-14), 144.1,129.7$ (C-10), 129.5 (C-12), 127.0, 116.4, 114.5, 113.2, 92.2, 57.6, 57.3, 56.9; IR $\left(\mathrm{KBr}, \mathrm{cm}^{-1}\right)$ Vmax: 2942, 1605, 1515, 1271, 1128; Elemental Analysis Anal. calcd for $\mathrm{C}_{19} \mathrm{H}_{19} \mathrm{FO}_{5}$ : C, 65.89; H, 5.53; Found: C, 65.55; H, 5.46.

(E)-3-(2,5-Difluoro-4-methoxyphenyl)-1-(2,4,6-trimethoxyphenyl)prop-2-en-1-one (14): Crude product was precipitated with (DCM/Hexane). Final product yellow solid; yield 70\%; $\mathrm{R}_{f}=($ EtOAc/Hexane 30:70) $=0,33$; m.p. $176-178{ }^{\circ} \mathrm{C} ;{ }^{1} \mathrm{H}-\mathrm{NMR}\left(400 \mathrm{MHz}, \mathrm{CDCl}_{3}\right) \delta 7.43(\mathrm{~d}, J=16.0 \mathrm{~Hz}, 1 \mathrm{H}), 7.29-7.24(\mathrm{~m}, 1 \mathrm{H})$, $6.86(\mathrm{~d}, J=16.4 \mathrm{~Hz}, 1 \mathrm{H}), 6.69-6.65(\mathrm{~m}, 1 \mathrm{H}), 6.15(\mathrm{~s}, 2 \mathrm{H}), 3.89(\mathrm{~s}, 3 \mathrm{H}), 3.85(\mathrm{~s}, 3 \mathrm{H}), 3.77(\mathrm{~s}, 6 \mathrm{H}) ;{ }^{13} \mathrm{C}-$ NMR $\left(100 \mathrm{MHz}, \mathrm{CDCl}_{3}\right) \delta 193.8,162.8,159.2,157.9\left(\mathrm{dd}, \mathrm{C}-16, J_{C F}=256.2,2.2 \mathrm{~Hz}\right), 148.9(\mathrm{dd}, \mathrm{C}-13$, $\left.J_{C F}=242.1,2.8 \mathrm{~Hz}\right), 134.9,131.8\left(\mathrm{dd}, \mathrm{C}-14, J_{C F}=5.8,1.5 \mathrm{~Hz}\right), 129.9,114.5\left(\mathrm{dd}, \mathrm{C}-12, J_{C F}=20.5,4.9\right.$ $\mathrm{Hz}), 111.9,106.5\left(\mathrm{dd}, \mathrm{C}-11, J_{C F}=24.4,2,8 \mathrm{~Hz}\right), 101.7\left(\mathrm{dd}, \mathrm{C}-15, J_{C F}=28.4,1.8 \mathrm{~Hz}\right), 91.0,56.7,56.1$, 55.7; IR (KBr, $\left.\mathrm{cm}^{-1}\right)$ Vmax: 2941, 1604, 1516, 1457, 1227, 1128; Elemental Analysis Anal. calcd for $\mathrm{C}_{19} \mathrm{H}_{18} \mathrm{~F}_{2} \mathrm{O}_{5}$ : C,62.63; H,4.98; Found: C,62.50; H,4.62.

\subsubsection{General Procedure for Preparation of Compounds $(\mathbf{9}, 11,13)$} of $\mathrm{MeOH}$.

The general procedure described in 2.2.1 was applied using benzaldehydes $\mathbf{4 , 5 , 6}$ in THF instead

(E)-3-(2,4-Difluorophenyl)-1-(2,4,6-trimethoxyphenyl)prop-2-en-1-one (9): Crude product was precipitated with $(\mathrm{DCM} / \mathrm{Hexane})$. Final product yellow solid; yield $75 \% ; \mathrm{R}_{f}=($ EtOAc/Hexane 30:70) $=$ 0.4; m.p. 129-131 ${ }^{\circ} \mathrm{C}$; ${ }^{1} \mathrm{H}-\mathrm{NMR}\left(400 \mathrm{MHz}, \mathrm{CDCl}_{3}\right) \delta 7.55$ (dd, $\left.J=14.9,6.7 \mathrm{~Hz}, 1 \mathrm{H}\right), 7.46(\mathrm{~d}, J=16.3$ $\mathrm{Hz}, 1 \mathrm{H}), 6.96(\mathrm{~d}, J=16.2 \mathrm{~Hz}, 1 \mathrm{H}), 6.90-6.80(\mathrm{~m}, 2 \mathrm{H}), 6.14(\mathrm{~s}, 2 \mathrm{H}), 3.84(\mathrm{~s}, 3 \mathrm{H}), 3.76(\mathrm{~s}, 6 \mathrm{H}) ;{ }^{13} \mathrm{C}-\mathrm{NMR}$ $\left(100 \mathrm{MHz}, \mathrm{CDCl}_{3}\right) \delta 195.1,165.5\left(\mathrm{dd}, \mathrm{C}-14, J_{C F}=224,12.3 \mathrm{~Hz}\right), 164.06,162.8\left(\mathrm{dd}, \mathrm{C}-16, J_{C F}=227\right.$, $11.7 \mathrm{~Hz}), 160.4,136.4,132.2,131.5\left(\mathrm{~d}, \mathrm{C}-12, J_{C F}=9.9 \mathrm{~Hz}\right), 121.0\left(\mathrm{~d}, \mathrm{C}-10, J_{C F}=11.5 \mathrm{~Hz}\right), 113.4(\mathrm{~d}, \mathrm{C}-$ $\left.13, J_{C F}=21.7 \mathrm{~Hz}\right), 112.9,105.9\left(\mathrm{~d}, \mathrm{C}-15, J_{C F}=25.3 \mathrm{~Hz}\right), 92.2,57.3,56.9 ; \mathrm{IR}\left(\mathrm{KBr}, \mathrm{cm}^{-1}\right)$ Vmax: 2941 , 1654, 1606, 1128; Elemental Analysis Anal. calcd for $\mathrm{C}_{18} \mathrm{H}_{16} \mathrm{~F}_{2} \mathrm{O}_{4}$ : C, 64.67; H, 4.82; Found: C, 64.22; $\mathrm{H}, 4.83$.

(E)-3-(3,4-Difluorophenyl)-1-(2,4,6-trimethoxyphenyl)prop-2-en-1-one (11): Crude product was precipitated with $(\mathrm{DCM} /$ Hexane $)$. Final product yellow solid; yield $81 \% ; \mathrm{R}_{f}=($ EtOAc/Hexane 30:70) $=$ 0.33; m.p. 115-117 ${ }^{\circ} \mathrm{C} ;{ }^{1} \mathrm{H}-\mathrm{NMR}\left(400 \mathrm{MHz}, \mathrm{CDCl}_{3}\right) \delta 7.37-7.28(\mathrm{~m}, 1 \mathrm{H}), 7.25-7.20(\mathrm{~m}, 2 \mathrm{H}), 7.18-$ $7.10(\mathrm{~m}, 1 \mathrm{H}), 6.86(\mathrm{~d}, J=16.0 \mathrm{~Hz}, 1 \mathrm{H}), 6.15(\mathrm{~s}, 2 \mathrm{H}), 3.85(\mathrm{~s}, 3 \mathrm{H}), 3.77(\mathrm{~s}, 6 \mathrm{H}) ;{ }^{13} \mathrm{C}-\mathrm{NMR}(100 \mathrm{MHz}$, $\left.\mathrm{CDCl}_{3}\right) \delta 194.8,164.1,160.4,152.8\left(\mathrm{dd}, \mathrm{C}-15, J_{C F}=251.3,13.1 \mathrm{~Hz}\right), 151.9\left(\mathrm{dd}, \mathrm{C}-14, J_{C F}=247,13 \mathrm{~Hz}\right)$, $142.49,133.7(\mathrm{C}-10), 131.2,126.4(\mathrm{C}-12), 119.1\left(\mathrm{~d}, \mathrm{C}-14, J_{C F}=17.6 \mathrm{~Hz}\right), 117.9$ (d, C-16, $\left.J_{C F}=17.5 \mathrm{~Hz}\right)$, 113.0, 92.2, 57.3, 56.9; IR $\left(\mathrm{KBr}, \mathrm{cm}^{-1}\right)$ Vmax: 2940, 1604, 1515, 1266, 1128; Elemental Analysis Anal. calcd for $\mathrm{C}_{18} \mathrm{H}_{16} \mathrm{~F}_{2} \mathrm{O}_{4}$ : C, 64.67; H, 4.82; Found: C, 64.53; H, 4.83.

(E)-3-(2,4,5-Trifluorophenyl)-1-(2,4,6-trimethoxyphenyl)prop-2-en-1-one (13): Crude product was precipitated with $(\mathrm{DCM} / \mathrm{Hexane})$. Final product yellow solid; yield $74 \% ; \mathrm{R}_{f}=($ EtOAc/Hexane 30:70) $=$ 0.4; m.p. 109-111 ${ }^{\circ} \mathrm{C} ;{ }^{1} \mathrm{H}-\mathrm{NMR}\left(400 \mathrm{MHz}, \mathrm{CDCl}_{3}\right) \delta 7.50-7.35(\mathrm{~m}, 2 \mathrm{H}), 7.02-6.91(\mathrm{~m}, 2 \mathrm{H}), 6.17$ (s, 2H), $3.88(\mathrm{~s}, 3 \mathrm{H}), 3.80(\mathrm{~s}, 6 \mathrm{H}) ;{ }^{13} \mathrm{C}-\mathrm{NMR}\left(100 \mathrm{MHz}, \mathrm{CDCl}_{3}\right) \delta 193.3,163.0,159.4,156.8\left(\mathrm{dd}, \mathrm{C}-16, J_{C F}\right.$ $=249.4,9.7 \mathrm{~Hz}), 151.2\left(\mathrm{dd}, \mathrm{C}-13, J_{C F}=254.1,12.8 \mathrm{~Hz}\right), 147.3\left(\mathrm{dd}, \mathrm{C}-14, J_{C F}=244.5,16.1 \mathrm{~Hz}\right), 133.6$, $131.8,120.1(\mathrm{C}-10), 116.2$ (ddd, C-12, $\left.J_{C F}=15.9,11.6,4.3 \mathrm{~Hz}\right), 111.6,106.4$ (ddd, C-15, $J_{C F}=28.4$, 21.3, $11 \mathrm{~Hz}), 90.9,56.1,55.6$; IR $\left(\mathrm{KBr}, \mathrm{cm}^{-1}\right)$ Vmax: 2943, 1605, 1508, 1129; Elemental Analysis Anal. calcd for $\mathrm{C}_{18} \mathrm{H}_{15} \mathrm{~F}_{3} \mathrm{O}_{4}$ : C, 61.37; H, 4.29; Found: C, 61.59; H,4.27.

\section{Results and Discussion}

Although new synthetic methods have been developed for the synthesis of chalcones, the basecatalyzed Claisen-Schmidt condensation reaction is one of the most common methods used to obtain chalcones. In this study, commercially available fluorine-substituted benzaldehydes (2-6) and 2',4',6'Trimethoxyacetophenone (1) were treated with $\mathrm{KOH}$, and using Claisen-Schmidt condensation, the synthesis of chalcones containing $2^{\prime}, 4^{\prime}, 6^{\prime}$-trimethoxy in the ring A, and chalcones containing only fluorine or fluorine-methoxy substituted groups in the ring B (7-14) were achieved. 


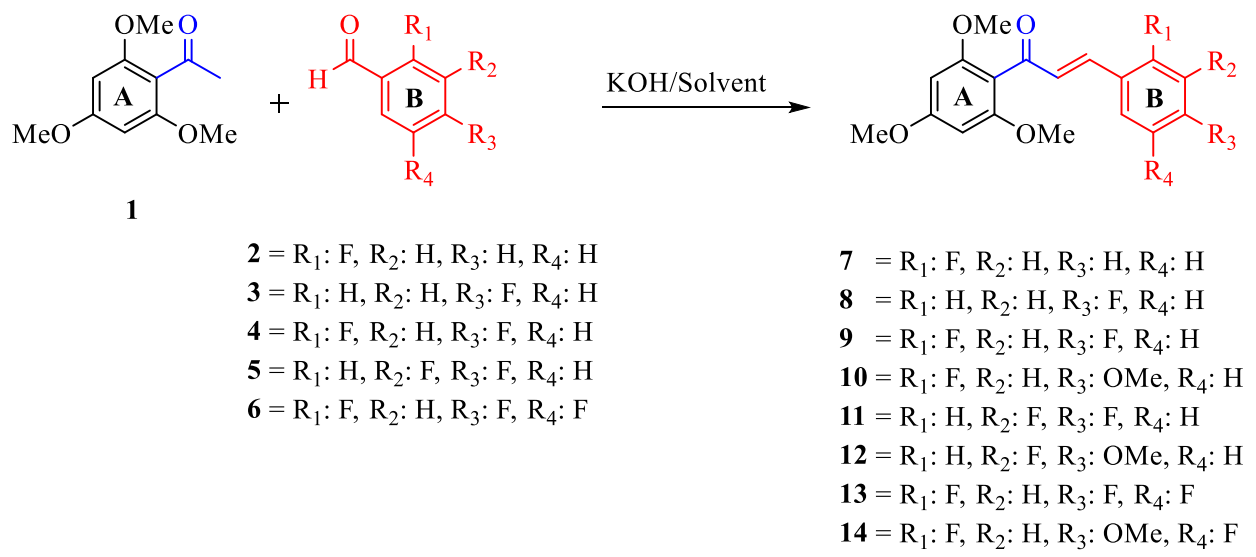

Scheme 2. General synthesis method

In the literature, in the synthesis of chalcones using Claisen-Schmidt condensation reactions, usually $\mathrm{KOH}$ is used as a base and $\mathrm{MeOH}$ is used as a reaction solvent. ${ }^{18}$ The nucleophilic property of $\mathrm{MeOH}$ is increased in a basic condition. The fluoro-benzaldehydes (2-6) selected in this study have the aldehyde group (CHO), which has both fluorine and electron withdrawing group in the aromatic $\mathrm{B}$ ring. In this study, a series of reaction experiments were performed not only for fluorine-containing chalcone derivatives $7,8,9,11,13$ but also for the synthesis of fluoro-chalcone derivatives 10, 12, 14 that contain para methoxy substituents as a result of replacement of fluorine groups with the methoxy group, whose nucleophilicy increases in the basic condition, through a nucleophilic aromatic substitution reaction $\left(\mathrm{S}_{\mathrm{N}} \mathrm{Ar}\right)$ (Scheme 2).

In the first stage, optimization experiments were performed for the reaction of 2,4,5trifluorobenzaldehyde (6) with output compound $2^{\prime}, 4^{\prime}, 6^{\prime}$-trimethoxyacetophenone (1) in $\mathrm{MeOH}$ accompanied by $\mathrm{KOH}$. No conversion was observed in the reaction under conditions using $\mathrm{KOH} 1$ equivalent (Table 1, entry1). When $\mathrm{KOH}$ was used 5 molar equivalent, the compound $\mathbf{1 4}$ was obtained.

Table 1. Optimization of the reaction conditions for the formation of chalcones 13 and $14 .^{[a]}$<smiles>COc1cc(OC)c(C(C)=O)c(OC)c1</smiles><smiles>COc1cc(OC)c(C(=O)/C=C/c2cc(F)c(F)cc2F)c(OC)c1</smiles><smiles>COc1cc(OC)c(C(=O)/C=C/c2cc(OC)c(F)cc2F)c(OC)c1</smiles>

\begin{tabular}{ccccc}
\hline Entry & Base $\mathbf{( 5 0 \%}$ KOH $\left._{(\mathbf{a q})}\right){\text { [equiv. }]^{[\mathbf{b}]}}$ & Solvent & Product & Yield [\%] $^{[\mathbf{c}]}$ \\
\hline 1 & 1 molar eq. & $\mathrm{MeOH}$ & No reaction & - \\
2 & 5 molar eq. & $\mathrm{MeOH}$ & $\mathbf{1 4}$ & 70 \\
3 & 15 molar eq. & $\mathrm{MeOH}$ & $\mathbf{1 4}$ & 60 \\
4 & 45 molar eq. & $\mathrm{MeOH}$ & $\mathbf{1 4}$ & 55 \\
5 & 60 molar eq. & $\mathrm{MeOH}$ & $\mathbf{1 4}$ & 50 \\
6 & 5 molar eq. & $\mathrm{THF}$ & $\mathbf{1 3}$ & 74 \\
\hline
\end{tabular}

[a] A solution of 1 (1 molar eq.), benzaldehyde $\mathbf{6}$ (1.6 molar eq and $\mathrm{KOH}$ ([b] different eq.) in solvent $(1 \mathrm{~mL})$ was stirred overnight at room temperature. After full conversion of acetophenone 1, the reaction was quenched with $\mathrm{NH}_{4} \mathrm{Cl}_{(\mathrm{aq})}$. [c] Isolated yield. 
As mentioned earlier, it is clear that the fluorine at the para position of the aromatic ring to which three fluorines are bound and where the methoxy groups are easily replaced through nucleophilic aromatic substitution reaction $\left(\mathrm{S}_{\mathrm{N}} \mathrm{Ar}\right)$.

The compound $\mathbf{1 3}$ was not observed in this reaction condition, while it was determined that the reaction efficiency of isolated $\mathbf{1 4}$ was $70 \%$ yield (Table 1, entry 2). Later, when the amount of $\mathrm{KOH}$ was increased to improve the reaction efficiency, some decomposition products were formed in addition to $\mathbf{1 4}$ in the reactions followed by ${ }^{1} \mathrm{H}-\mathrm{NMR}$. As a result of this situation, the reaction efficiency for $\mathbf{1 4}$ was found to be decreased (Table 1, entry 3-5). From these results, it was concluded that if $\mathrm{MeOH}$ was used as a solvent in a condensation reaction with $\mathbf{6}$ comprising three fluorine, the fluorine in the para position was easily replaced by the methoxy group by a $S_{N} A r$ reaction.

At this point, it was planned to compare the previous results with the results to be obtained by using a different solvent in lieu of $\mathrm{MeOH}$ as a reaction solvent. Considering the optimized reaction conditions in the previous experiments, the most suitable solvent for the reaction mixture in the basic condition was thought to be tetrahydrofuran (THF). In the condensation reaction performed with $\mathrm{KOH}$ in THF under optimized reaction conditions, the compound $\mathbf{1 3}$ was obtained with a $74 \%$ yield (Table 1, entry 6).

In the next stage of the study, experiments were conducted using different fluorine substituted benzaldehydes $(\mathbf{2}, \mathbf{3}, \mathbf{4}, \mathbf{5})$ in order to determine whether $\mathrm{S}_{\mathrm{N}} \mathrm{Ar}$ reaction would occur at different positions in the aromatic $\mathrm{B}$ ring and was dependent on the number of fluorine.

Table 2. Optimization of the reaction conditions for the formation of chalcone $\mathbf{7}$ and $\mathbf{8}{ }^{\left[{ }^{[a]}\right.}$

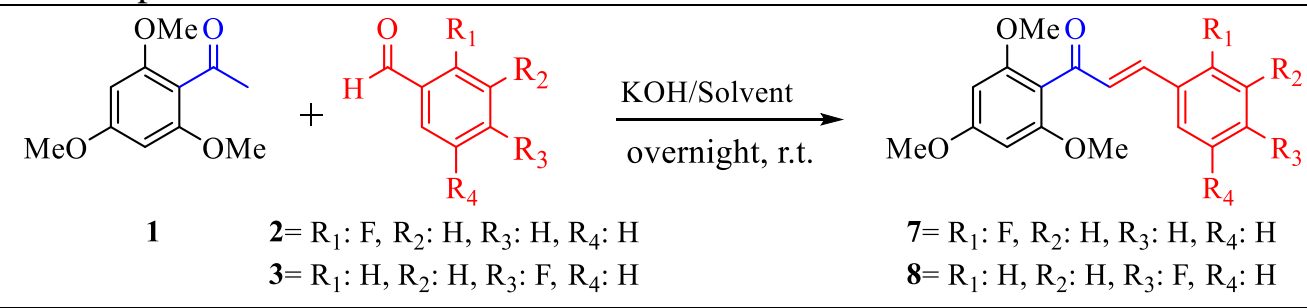

\begin{tabular}{|c|c|c|c|c|c|}
\hline Entry & $\begin{array}{c}\text { Base }(50 \% \text { KOH }) \\
\text { [equiv] }^{\text {bb] }}\end{array}$ & Solvent & $\begin{array}{c}\text { Fluoro - } \\
\text { Benzaldehyde }\end{array}$ & Product & $\begin{array}{l}\text { Yield } \\
{[\%]^{[\mathrm{c}]}}\end{array}$ \\
\hline 1 & 5 molar eq. & $\mathrm{MeOH}$ & 2 & 7 & 92 \\
\hline 2 & 15 molar eq. & $\mathrm{MeOH}$ & 2 & 7 & 82 \\
\hline 3 & 60 molar eq. & $\mathrm{MeOH}$ & 2 & 7 & 82 \\
\hline 4 & 5 molar eq. & THF & 2 & 7 & 90 \\
\hline 5 & 5 molar eq. & $\mathrm{MeOH}$ & 3 & 8 & 80 \\
\hline 6 & 15 molar eq. & $\mathrm{MeOH}$ & 3 & 8 & 80 \\
\hline 7 & 60 molar eq. & $\mathrm{MeOH}$ & 3 & 8 & 80 \\
\hline 8 & 5 molar eq. & THF & 3 & 8 & 85 \\
\hline
\end{tabular}

[a] A solution of $\mathbf{1}$ (1 molar eq.), 4-florobenzaldehyde 3 (1.6 molar eq), and KOH ( [b] different eq.) in solvent $(1 \mathrm{~mL})$ was stirred overnight at room temperature. After full conversion of acetophenone 1, the reaction was quenched with $\mathrm{NH}_{4} \mathrm{Cl}_{(\mathrm{aq})}$. [c] Isolated yield.

Particularly in experiments conducted in different amounts and solvents with para-fluorobenzaldehyde (3) comprising fluorine at the para position in the B ring, only the compound $\mathbf{8}$ was achieved with a high efficiency (Table 2, entries 5 and 8). When high base concentrations were reached, it was determined that the conversion to compound $\mathbf{8}$ and the reaction efficiency did not change (Table 2, entries 6 and 7). It was concluded that the $S_{N} A r$ reaction did not take place when there was only one fluorine in the aromatic ring B. As mentioned in the introduction, the low percentage of fluorine in the aromatic ring in such systems reduces $S_{N} A r$ efficiency. ${ }^{19}$ While no replacement product was found in the experiments conducted simultaneously with ortho-fluorobenzaldehyde (2) under same conditions, condensation product (7) was obtained with high efficiency (Table 2, entry 1-4). As a result of the absence of replacement, condensation efficiency increased and compound 7 was obtained with $92 \%$ yield (Table 2 , entry 1). 
Based on the systematic data obtained from the experiments conducted with one- and threemember fluorine-substituted derivatives of aromatic fluorine-substituted benzaldehydes $(\mathbf{2}, \mathbf{3}, \mathbf{6})$, similar studies with two-member derivatives $(\mathbf{4}, \mathbf{5})$ were made and the results shown in Table 3 were obtained.

Table 3. Optimization of the reaction conditions for the formation of chalcone $9,10,11$ and 12 . ${ }^{[a]}$<smiles>[R]c1cc(/C=C/C(=O)c2c(OC)cc(OC)cc2OC)c([R])c(/C=C/c2cc([R19])c([R3])c([R])c2[R])c1</smiles>

1

$$
\begin{aligned}
& \mathbf{4}=\mathrm{R}_{1}: \mathrm{F}, \mathrm{R}_{2}: \mathrm{H}, \mathrm{R}_{3}: \mathrm{F}, \mathrm{R}_{4}: \mathrm{H} \\
& \mathbf{5}=\mathrm{R}_{1}: \mathrm{H}, \mathrm{R}_{2}: \mathrm{F}, \mathrm{R}_{3}: \mathrm{F}, \mathrm{R}_{4}: \mathrm{H}
\end{aligned}
$$

\begin{tabular}{|c|c|c|c|c|c|}
\hline Entry & $\begin{array}{c}\text { Base }(50 \% \text { KOH) } \\
\text { [equiv] }^{[b]}\end{array}$ & Solvent & $\begin{array}{c}\text { Fluoro - } \\
\text { Benzaldehyde }\end{array}$ & Product & Yield $[\%]^{[c]}$ \\
\hline 1 & 5 molar eq. & $\mathrm{MeOH}$ & 4 & 9 and $10^{[\mathrm{d}]}$ & $35 / 50$ \\
\hline 2 & 15 molar eq. & $\mathrm{MeOH}$ & 4 & 9 and $10^{[\mathrm{d}]}$ & low yield/40 \\
\hline 3 & 60 molar eq. & $\mathrm{MeOH}$ & 4 & 9 and $10^{[\mathrm{d}]}$ & low yield/40 \\
\hline 4 & 5 molar eq. & THF & 4 & 9 & 75 \\
\hline 5 & 5 molar eq. & $\mathrm{MeOH}$ & 5 & 11 and $12^{[\mathrm{d}]}$ & $20 / 70$ \\
\hline 6 & 15 molar eq. & $\mathrm{MeOH}$ & 5 & 11 and $12^{[\mathrm{d}]}$ & low yield/60 \\
\hline 7 & 60 molar eq. & $\mathrm{MeOH}$ & 5 & 11 and $12^{[\mathrm{d}]}$ & low yield/60 \\
\hline 8 & 5 molar eq. & THF & 5 & 11 & 81 \\
\hline
\end{tabular}

$$
\begin{aligned}
& \mathbf{9}=\mathrm{R}_{1}: \mathrm{F}, \mathrm{R}_{2}: \mathrm{H}, \mathrm{R}_{3}: \mathrm{F}, \mathrm{R}_{4}: \mathrm{H} \\
& \mathbf{1 0}=\mathrm{R}_{1}: \mathrm{F}, \mathrm{R}_{2}: \mathrm{H}, \mathrm{R}_{3}: \mathrm{OMe}, \mathrm{R}_{4}: \mathrm{H} \\
& \mathbf{1 1}=\mathrm{R}_{1}: \mathrm{H}, \mathrm{R}_{2}: \mathrm{F}, \mathrm{R}_{3}: \mathrm{F}, \mathrm{R}_{4}: \mathrm{H} \\
& \mathbf{1 2}=\mathrm{R}_{1}: \mathrm{H}, \mathrm{R}_{2}: \mathrm{F}, \mathrm{R}_{3}: \mathrm{OMe}, \mathrm{R}_{4}: \mathrm{H}
\end{aligned}
$$

[a] A solution of $\mathbf{1}$ (1 molar eq.), 4-florobenzaldehyde $\mathbf{3}$ (1.6 molar eq.), and $\mathrm{KOH}$ ([b] different eq.) in solvent $(1 \mathrm{~mL})$ was stirred overnight at room temperature. After full conversion of acetophenone $\mathbf{1}$, the reaction was quenched with $\mathrm{NH}_{4} \mathrm{Cl}_{(\mathrm{aq})}$. [c] Isolated yield. [d] mixture of product in the same reaction condition.

In similar studies conducted with $\mathbf{4}$ and $\mathbf{5}$ fluoro-benzaldehydes, interestingly the relevant products 9-10 and 11-12 were observed in the reactions conducted with $\mathrm{MeOH}$ (Table 3, entries 1-3 and 5-7). This brought about the conclusion that the $\mathrm{S}_{\mathrm{N}} \mathrm{Ar}$ reaction occurred again in $\mathrm{MeOH}$, but the reaction rate was slow. When THF was used as a reaction solvent, only the relevant compounds $\mathbf{9}$ and $\mathbf{1 1}$ were isolated with high purity and efficiency as expected in experiments conducted with both fluorobenzaldehyde derivatives (4 and $\mathbf{5}$ ) (Table 3 , entry 4 and 8).

In these sequential experiments, which take place under basic conditions in $\mathrm{MeOH}$, the methoxy anion attacking the electron-poor aromatic ring $\mathrm{B}$ acts as a nucleophile. In nucleophilic aromatic substitution reactions, it is often noted as a leaving group when there is fluorine in the electron-poor ring. In the attack of nucleophile at the para position, the negative charge can be delocalized to the oxygen of the carbonyl group. The negatively charged intermediate is also stabilized by electron with drawing group via carbonyl group. It is clear that fluorine substituents increase the nucleophilic aromatic substitution rate. In addition, an increase in the number of highly electronegative fluorides in ring $\mathrm{B}$ helps activate the ring toward attack.

Based on these results, when there is only one Fluorine atom in aromatic ring B, there is no replacement of fluor with methoxy. Substitution only takes place where there is more than one fluorine atom and only at para position and only in $\mathrm{MeOH}$. These evidences indicate that more electron deficiency is provided with more fluorine atoms, otherwise no substitution takes place. Para position is understandable since that position is more susceptible. Moreover, the results indicate that sole presence of more fluorine atoms and para position are not enough for the substitution; the reaction should take place in a protic solvent like $\mathrm{MeOH}$. An explanation for that could be the hydrogen bonding with the carbonyl oxygen of the aldehyde, which makes the carbon atom of the para fluor more positive through conjugation, considering that Fluorine atom is the most electronegative atom and cannot donate its electrons as efficient as the other halogens. 


\section{Conclusion}

Base-catalyzed syntheses of chalcone derivatives $(10,11,12,13,14)$, whose syntheses were previously unknown in the literature, were synthesized efficiently and in high yields. For the synthesis of target products in this study, an effective method was developed using THF as a solvent instead of $\mathrm{MeOH}$, which is frequently used as a solvent in chalcone syntheses conducted in basic condition through ClaisenSchmidt condensation reactions. The experiments conducted in this study revealed that THF has to be determined as the reaction solvent when synthesis of relevant chalcone derivatives $(\mathbf{7}, \mathbf{8 , 9}, \mathbf{1 1}, \mathbf{1 3})$ with fluorine-substituted benzaldehydes (2-6) used as output compounds is intended.

On the other hand, in case $\mathrm{MeOH}$ was used as a reaction solvent, nucleophilic aromatic substitution reaction $\left(\mathrm{S}_{\mathrm{N}} \mathrm{Ar}\right)$ was determined to have occurred in the reaction condition. No substitution product was observed in the experiments conducted with fluorobenzaldehyde derivatives (2 and $\mathbf{3})$ containing only one fluorine-substituent. When the literature is examined, the syntheses of compounds $(7,8,9)$ in basic condition and in $\mathrm{MeOH}$ have previously been performed ${ }^{20-22}$ However, these compounds can be effectively and efficiently obtained in our reactions conducted in THF.

In cases where the number of fluorine in the aromatic ring is two or three, it was determined that fluorine in the para position is competitively replaced with methoxy. The chalcone derivatives $(\mathbf{1 0}, \mathbf{1 1}$, $12,13,14)$ obtained as a result of the reactions conducted in $\mathrm{MeOH}$ and THF were also isolated in high purity and new compounds unknown in the literature were obtained.

\section{Supporting Information}

Supporting information accompanies this paper on http://www.acgpubs.org/journal/organic$\underline{\text { communications }}$

\section{ORCID}

Derya Aktaş Anıl: 0000-0003-2584-275X

M. Fatih Polat: $\underline{0000-0002-2838-163 X}$

\section{References}

[1] Dolbier, W. R. Fluorine chemistry at the millennium. J. Fluor. Chem. 2005, 126(2), 157-163.

[2] Shah, P.; Westwell, A. D. The role of fluorine in medicinal chemistry. J. Enzym. Inhib. Med. Chem. 2007, 22(5), 527-540.

[3] O'Hagan, D. Understanding organofluorine chemistry. An introduction to the C-F bond. Chem. Soc. Rev. 2008, 37(2), 308-319.

[4] Uneyama, K. Fundamentals in Organic Fluorine Chemistry. In: Organofluorine Chemistry 2007, 1-100.

[5] Terrier, F. Modern Nucleophilic Aromatic Substitution. Wiley-VCH Verlag, 2013, 1-472.

[6] Kwan, E. E.; Zeng, Y.; Besser, H. A.; Jacobsen, E. N. Concerted nucleophilic aromatic substitutions. Nat Chem. 2018, 10(9), 917-923.

[7] Kirsch, P. Synthesis of Complex Organofluorine Compounds. In: Modern Fluoroorganic Chemistry. WileyVCH Verlag GmbH \& Co. KGaA, 2005, 25-169.

[8] Ni, C.; Hu, J. The unique fluorine effects in organic reactions: Recent facts and insights into fluoroalkylations. Chem. Soc. Rev. 2016, 45(20), 5441-5454.

[9] Bégué, J. P.; Bonnet-Delpon, D. Recent advances (1995-2005) in fluorinated pharmaceuticals based on natural products. J. Fluor. Chem. 2006, 127, 992-1012.

[10] Kirk, K. L. Fluorine in medicinal chemistry: Recent therapeutic applications of fluorinated small molecules. J. Fluor. Chem. 2006, 127, 1013-1029.

[11] Ojima, I. Fluorine in Medicinal Chemistry and Chemical Biology. John Wiley and Sons, 2009, 1-624.

[12] Dhar, D. N. The chemistry of chalcones and related compounds. Wiley, 1981, 285. 
[13] Lunardi, F.; Guzela, M.; Rodrigues, A. T.; Correa, R.; Eger-Mangrich, I.; Steindel, M.; Grisard, E.C.; Assreuy, J.; Calixto, J.B.; Santos A.R. Trypanocidal and leishmanicidal properties of substitution-containing chalcones. Antimicrob Agents Chemother. 2003, 47(4), 1449-1451.

[14] Dimmock, J. R.; Elias, D. W.; Beazely, M. A.; Kandepu, N. M. Bioactivities of chalcones. Curr. Med. Chem. 1999, 6(12), 1125-1149.

[15] Fandakl1, S.; Doğan, İ.S.; Sellitepe, H.E.; Yasar, A.; Yaylı, N. Synthesis, theoretical calculation and $\alpha-$ glucosidase inhibition of new chalcone oximes. Org. Commun. 2018, 11 (1),23-34.

[16] Ahcene, B.; Xavier, R.; Jean, B.; Boumendjel, A.; Ronot, X.; Boutonnat, J. Chalcones derivatives acting as cell cycle blockers: potential anti cancer drugs. Curr. Drug Targets 2009, 10(4), 363-371.

[17] Das, M.; Manna, K. Chalcone scaffold in anticancer Armamentarium: A molecular insight. J. Toxicol. 2016, $1-14$.

[18] Nielsen, A.; Houlihan, W. Aldol condesation. Org. React. 1968, 16, 1-438 https://doi.org/10.1002/ 0471264180.or016.01.

[19] Pažitný, A.; Solčán, T.; Végh, D. Pentafluorobenzaldehyde and its utilizing in organic synthesis. J. Fluor. Chem. 2009, 130, 267-294.

[20] Yalcin, G.; Burmaoglu, S.; Yildiz, I.; Algul, O. Molecular docking studies on fluoro-substituted chalcones as potential DprE1 enzyme inhibitors. J. Mol. Struct. 2018, 1164, 50-56.

[21] Thieury, C.; Lebouvier, N.; Guével, R.; Barguil, Y.; Herbette, G.; Antheaume, C.; Hnawia, E.; Asakawa, Y.; Nour,; Guillaudeux, T. Mechanisms of action and structure-activity relationships of cytotoxic flavokawain derivatives. Bioorg. Med. Chem. 2017, 25(6), 1817-1829.

[22] Burmaoglu, S.; Algul, O.; Gobek, A.; Aktas-Anil, D.; Ulger, M.; Erturk, B.G.,; Kaplan, E.; Dogen, A.; Aslan, G. Design of potent fluoro-substituted chalcones as antimicrobial agents. J. Enzym. Inhib. Med. Chem. 2017, $32(1), 490-495$.

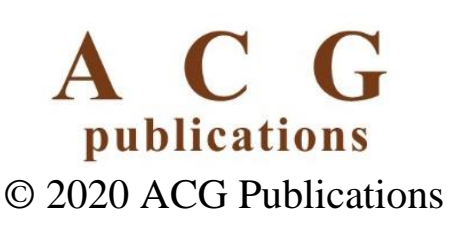

